\title{
Occult HBV infection
}

\author{
Mariangela Spinosa, Pasquale Spagnuolo \\ U.O. Medicina Trasfusionale-P.O. S.Timoteo Termoli (CB) Azienda Sanitaria Regionale Del Molise
}

Key words: HBV genome, HbsAg-negative, Occult infection

Infezione occulta da HPV

\section{SUMMARY}

The presence of hepatitis B virus genome in HBsAg-negative subjects is known as "occult infection" from HBV. Patients with occult infection may express markers of previous exposure to B virus (anti-HBs and / or anti-HBc positive). $20 \%$ is negative for all $\mathrm{HBV}$ markers except for the presence of HBV-DNA. Occult infection is associated with a strong suppression of viral replication that is responsible for both $\mathrm{HBs} A g$ negativity and the very low or undetectable HBV-DNA in serum but seen in the liver tissue. The pathogenesis of occult infection is considered multifactorial. Infection by mutants of HBV (HBsAg mutants), suppression of viral replication and expression of genes of HBV, HBV-DNA integration into the host genome, infection of HBV in mononuclear peripheral blood, abnormalities of the immune response, interference with other virus. HBV DNA with high sensitivity and specificity, can shorten the "window period" elapses between exposure to infection and responsiveness to the serological test. The presence of mutant HBV amino acid substitution of the determinant "a" gene " $S$ " epitope of the surface protein of the virus, causes conformational changes that alter the binding with the specific antibody.

Aim of this work is to assess negative or borderline subjects of $\mathrm{HbsAg}$, with moderate increases of transaminase levels but - positive to HBV DNA test. The serological markers of HBV were determined with Architect (Abbott). The HBV-DNA was evaluated in Real-Time PCR Cobas TaqMan 48 (Roche) a quantitative test that detects the viral concentration. I5350.12 (0.07\%) were positive for HBV-DNA test., 3 positive for $\mathrm{HBsAg}, 9$ negative HBV markers showed anti-HBc reactivity in all 12 anti-HBe in 3,4 anti-HBs with a titer of I3Ul/ml.Le ALT were altered only for 2 to 12 and was detected viremia below the sensitivity test $(<6 \mathrm{IU} / \mathrm{ml})$. These data allow to draw some considerations:

- Screening tests for infection with HBV do not show the "window period". The slow replication of HBV with low viremia in the initial phase involves the impossibility of identifying $\mathrm{HBsAg}$ in serological screening, but detected by molecular biology tests.

- The "window period", the carrier state immunosilent chronic, low levels of viral proteins do not detect the infection.

- Data in literature indicate the presence of different mutant strains of HBV characterized by altered expression of the antigenic determinant "a" of $\mathrm{HbsAg}$.

- The majority of screening tests for detection of HBsAg using monoclonal antibodies for the identification of the determinant "a" is needed to identify the different mutants using a mixture of monoclonal antibodies capable of recognizing even the most conserved regions. The gold standard methods to detect the mutant viruses are the techniques of molecular biology and the direct sequencing.

\section{INTRODUZIONE}

La presenza del genoma del virus dell'epatite B in soggetti HBsAg negativi è nota come "infezione occulta" da HVB.

Soggetti con infezione occulta possono avere marcatori di pregressa esposizione al virus B (anti-HBs e/o anti-HBc positivi). Il $20 \%$ risulta negativi per tutti i marcatori di HBV tranne che per la presenza di HBV-DNA.

L'infezione occulta è associata a una forte soppressione della replicazione virale che è responsabile sia della negatività dell'HBsAg sia del livello molto basso o non rilevabile di HBV-DNA nel siero ma evidenziabile nel tessuto epatico. La patogenesi dell'infezione occulta si ritiene multifattoriale: infezione da mutanti del virus HBV (mutanti di HBsAg), soppressione della replica virale e dell'espressione dei geni di HBV, integrazione di HBVDNA nel genoma dell'ospite, infezione di HBV nei mononucleati del sangue periferico, alterazioni della risposta immune, interferenza con altri virus. L'HBV DNA grazie all'elevata sensibilità e specificità, permette di abbreviare il "periodo finestra" intercorrente tra l'esposizione all'infezione e la reattività al test sierologico.

La presenza di mutanti dell'HBV la sostituzione amminoacidica del determinante "a" del gene "S", epitopo della proteina di superficie del virus, determina alterazioni conformazionali che alterano il legame con gli anticorpi specifici.

Scopo del nostro lavoro è valutare soggetti negativi o bor- derline all'HBsAg, con moderati rialzi delle transaminasi, ma positivi al test HBV-DNA.

\section{MATERIALI E METODI}

I marcatori sierologici di HBV sono stati eseguiti con Architect (Abbott). L'HBV-DNA in Real-Time PCR su Cobas TaqMan 48 (Roche) un test quantitativo che rileva la carica virale.

\section{RISULTATI E DISCUSSIONE}

Su 15350, 12 (0.07\%) risultano positivi al test HBV-DNA., 3 positivi per l'HBsAg, 9 negativi. I marcatori HBV hanno evidenziato reattività anti-HBc in tutti i 12 anti-HBe in 3, 4 antiHBs con un titolo di $13 \mathrm{UI} / \mathrm{ml}$. Le ALT risultavano alterate solo per 2 . Su 12 è stata rilevata una viremia inferiore alla sensibilità del test (<6 UI/ml) (Tabella 1$)$.

Questi dati permettono di trarre alcune considerazioni: - i test di screening per l'infezione da HBV non evidenziano il "periodo finesta". La lenta replicazione del virus HBV con bassa viremia nella fase iniziale comporta l'impossibilità di identificare l'HBsAg nello screening sierologico, ma evidenziabile mediante i test di biologia molecolare.

- Il "periodo finestra", lo stato di portatore cronico immunosilente, bassi livelli di proteine virali non rilevano l'infezione.

- I dati in letteratura indicano la presenza di diversi ceppi mutanti del virus HBV caratterizzati da un alterata espres-

Tabella I. Donatori positivi per l'HBV-DNA e profilo sierologico

\begin{tabular}{|c|c|c|c|c|c|}
\hline DONATORI & HBV-DNA & HBS-Ag & Anti-HBc & Anti-HBe & Anti-HBsAb \\
\hline 15350 & $12(0.07 \%)$ & 3 Positivi & I2 Positivi & 3 Positivi & 4 Positivi \\
\hline & $<6 \mathrm{Ul} / \mathrm{ml}$ & 3 Negativi & & & $13 \mathrm{Ul} / \mathrm{ml}$ \\
\hline
\end{tabular}

\section{Corresponding author: Mariangela Spinosa}

U.O. Medicina Trasfusionale-P.O. S.Timoteo Termoli (CB) Azienda Sanitaria Regionale Del Molise

Via Del Molinello, I - 86039 Campobasso

E-mail: mariangelaspi@virgilio.it 
sione antigenica del determinante “a” dell’HbsAg.

- La maggior parte dei test di screening per la rilevazione dell'HBsAg utilizza anticorpi monoclonali per l'identificazione del determinante" a", è necessario per poter identificare i diversi mutanti utilizzare una miscela di anticorpi monoclonali capaci di riconoscere anche le regioni più conservate. I metodi gold standard per rilevare i virus mutanti sono le tecniche di biologia molecolare e il sequenziamento diretto.

\section{BIBLIOGRAFIA}

1. Blum HE, Offensperger WB, Walter E, et al. Hepatitis B virus infection with full-length viral genome in a patient serollogically immune to hepatitis B infection. Liver Lancet 1988; 8: 307-16.

2. Manson AL, Xu L, Guo L, et al. Molecular basis foe persistent hepatitis Binfection in the liver after clearance of serum hepatis b surface antigen. Hepatology 1988; 27: 1736-42.

3. Tobenson M, Thomas DL. Occult hepatitis B. Lancet Infect Dis 2002; 2: 479-86. 